\title{
Exploiting nonlinearities of micro-machined resonators for filtering applications
}

S. Ilyas, K. N. Chappanda, and M. I. Younis

Citation: Appl. Phys. Lett. 110, 253508 (2017); doi: 10.1063/1.4986921

View online: http://dx.doi.org/10.1063/1.4986921

View Table of Contents: http://aip.scitation.org/toc/apl/110/25

Published by the American Institute of Physics

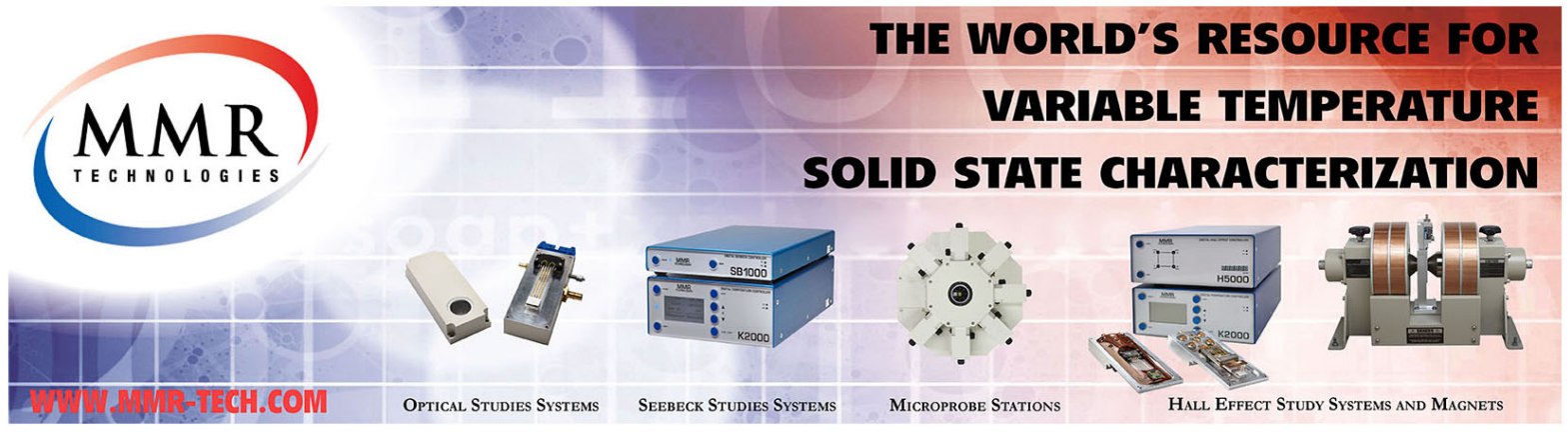




\title{
Exploiting nonlinearities of micro-machined resonators for filtering applications
}

\author{
S. Ilyas, K. N. Chappanda, and M. I. Younis ${ }^{a)}$ \\ Physical Science and Engineering Division, King Abdullah University of Science and Technology, \\ Thuwal 23955-6900, Saudi Arabia
}

(Received 29 March 2017; accepted 7 June 2017; published online 21 June 2017)

\begin{abstract}
We demonstrate the exploitation of the nonlinear behavior of two electrically coupled microbeam resonators to realize a band-pass filter. More specifically, we combine their nonlinear hardening and softening responses to realize a near flat pass band filter with sharp roll-off characteristics. The device is composed of two near identical doubly clamped and electrostatically actuated microbeams made of silicon. One of the resonators is buckled via thermal loading to produce a softening frequency response. It is then further tuned to create the desired overlap with the second resonator response of hardening behavior. This overlapping improves the pass band flatness. Also, the sudden jumps due to the softening and hardening behaviors create sharp roll-off characteristics. This approach can be promising for the future generation of filters with superior characteristics. Published by AIP Publishing. [http://dx.doi.org/10.1063/1.4986921]
\end{abstract}

Exploiting the nonlinear dynamics of micro and nano resonators for solving practical problems has been an intriguing area of research over the past two decades. The nonlinear behavior of microelectromechanical systems (MEMS) devices has been vastly studied and used for various applications including mechanical computing, ${ }^{1,2}$ wireless communication, ${ }^{3}$ and sensors and actuators. ${ }^{4}$ Similarly, the nonlinear behavior of nanoelectromechanical systems (NEMS) has gained considerable attention in the recent years. Considerable research is being conducted toward understanding the nonlinear dynamics of nanobeams, ${ }^{5}$ nanotubes, nanowires, and graphene based resonators. ${ }^{6}$ Moreover, various dynamical aspects in NEMS resonators, such as bifurcations, ${ }^{7}$ nonlinear damping, ${ }^{8}$ and chaos, ${ }^{9}$ are studied to understand their characteristics and utilize them for real life applications.

There has also been active research on the improvement of various aspects of MEMS resonators for frequency filtering applications, such as producing high-Q, high-frequency, and highly selective filters. ${ }^{10-29}$ Both electrically and mechanically coupled resonators have been demonstrated for filter applications. ${ }^{10-17}$ Highly tunable filters were studied, where the centre frequency and the bandwidth can be tuned independently. ${ }^{18-23}$ Furthermore, various designs of resonators have been explored, such as ring shaped and rectangular plate resonators, to achieve high-Q high-frequency filter operation. ${ }^{24-26}$ Parametric resonances, ${ }^{27,28}$ subharmonic excitations, ${ }^{29,30}$ and nonlinear feedback control ${ }^{31}$ have also been exploited. Multifunction MEMS devices, capable of performing several operations in a communication chain like simultaneous mixing/ filtering, have also been explored for energy efficient communication units. ${ }^{32,33}$

In this work, we present a technique that combines the hardening and softening responses of two electrically coupled resonators to demonstrate a near flat pass band and sharp roll-off MEMS filter. Toward this, electrothermal tuning and electrostatic actuation are used for two laterally

\footnotetext{
a)Mohammad.Younis@kaust.edu.sa
}

actuated doubly clamped microbeams. A schematic of one of the clamped-clamped beams along with the cross-sectional view of the physical layers comprising the structure is shown in Figs. 1(a) and 1(b). Figure 1(c) illustrates the experimental setup used to characterize the filter response.

The proposed filter consists of two nearly identical laterally actuated clamped-clamped microbeams. Each microbeam is anchored from its two ends with two fixed electrodes. An electrical current is passed through one of the beams via these electrodes for electrothermal tuning. The microbeams are actuated electrostatically with a driving electrode on the one side, and the output response is measured via a sensing electrode on the other side. The microbeam resonators are fabricated through a two-mask surface micromachining process using a highly conductive (resistivity of $0.001 \mathrm{ohm} \mathrm{cm}$ ) pdoped silicon on insulator (SOI) wafer $^{34}$ [see Fig. 1(b)]. The silicon layer is patterned, etched, and released to form the microbeam structure. A vector network analyzer (VNA) is used to characterize the resonators and demonstrate the filter operation [see Fig. 1(c)]. A single power supply is used to provide the same DC bias to both resonators. The radio frequency (RF) output from the network analyzer is applied to the drive electrodes of both beams. The output from the resonators are fed into the low noise amplifier (LNA), where they are combined and differentially amplified, and then sent back to the VNA. The outputs of these electrically coupled resonators can be combined using a differential-to-single ended amplifier ${ }^{21,35}$ (similar to the way the LNA is configured in the test setup) and interfaced with integrated circuits for the realization of the proposed filter in practical applications. A separate power supply is used to provide electrothermal voltage " $\mathrm{V}_{\mathrm{T}}$ " to one of the beams for frequency tuning. All the experiments are performed in moderate vacuum conditions at 1 Torr and at room temperature.

Figure 2 shows a schematic illustrating the basic principle of the proposed device. The responses of two resonators, one is of softening nonlinearity while the other is of hardening nonlinearity, are combined. Due to the nonlinear sudden 
(a)

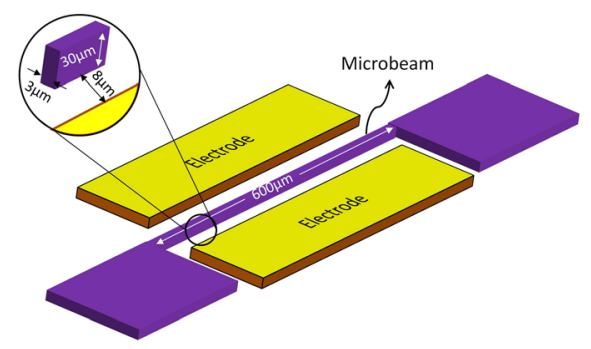

(c) (b)

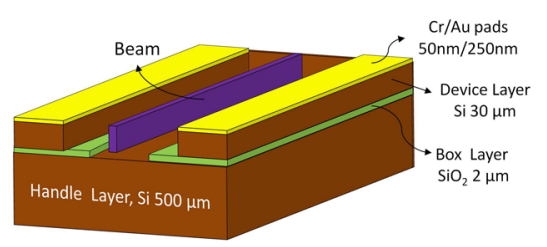

FIG. 1. (a) Schematic of the clampedclamped microbeam resonator. (b) $\mathrm{A}$ cross-section showing the various layers of the fabricated microbeam. The beam is fabricated on an SOI wafer with a 2 mask surface micromachining process. (c) Experimental setup for demonstrating the filter operation.

jumps at both sides of the combined response, sharp roll-off from the pass bands to the stop bands is achieved. The resonance frequency of one of the resonators is then electrothermally tuned to create a flat pass band. The combination of hardening and softening behaviors can be achieved, for instance, by using a cantilever that exhibits softening behavior and a clamped-clamped beam which shows hardening behavior. In this study, however, we utilize two clamped-clamped beams and rely on the electrothermal tuning of resonance frequencies of the beams to yield the desired filter characteristics. Hence, one beam is actuated beyond buckling to produce the quadratic nonlinearity for the softening behavior. This quadratic nonlinearity is of geometric type due to the new resulting configuration from buckling. The other beam is actuated below buckling such that the cubic nonlinearity is dominant. ${ }^{4,36}$ The cubic nonlinearity is a result of the beam midplane stretching generated at moderate deflection levels.

Using electrothermal voltage is beneficial as it allows for bidirectional large frequency tuning. ${ }^{36}$ This can be used to compensate for fabrication imperfections considering that the operating frequencies of the resonators are critical to the proposed technique for filtering.
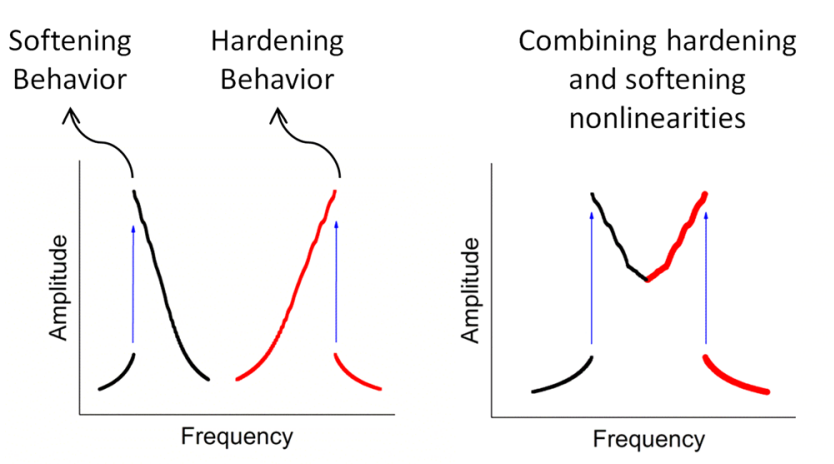

Nearly ideal filter

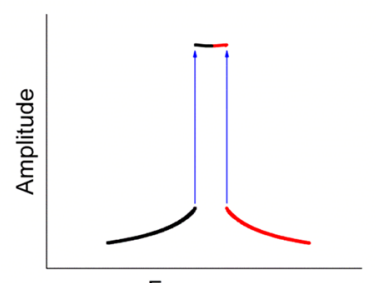

Frequency
Figure 3 shows an example of the obtained combined frequency response of the resonators. We notice that both beams exhibit hardening behavior as expected from a typical doubly clamped microbeam. The beams are designed to be of same dimensions; however, small fabrication imperfections cause a slight change in the dimensions and hence the resonance frequencies of the beams are slightly apart. Next, $\mathrm{V}_{\mathrm{T}}$ is applied to beam 1 to tune its frequency. Figure 4 shows the response of the resonator to various electrothermal loadings. We notice that as $\mathrm{V}_{\mathrm{T}}$ is increased, the resonance frequency of beam 1 decreases [see Fig. 4(a)]. This is due to the induced compressive axial load as a result of the current flow through the microbeam (Joule's heating). However, if $\mathrm{V}_{\mathrm{T}}$ is further increased, the microbeam buckles eventually, after which any increase in $\mathrm{V}_{\mathrm{T}}$ causes an increase in the resonance frequency of the microbeam ${ }^{36}$ [see Fig. 4(b)]. More importantly, the resonator nonlinearity becomes mainly of softening type, which is needed for the proposed filter concept. We observe that the responses of the two resonators start to overlap with each other at this point. By selecting a proper electrothermal voltage, an overlap is achieved giving the desired filter characteristics. A near flat pass band is possible due to
FIG. 2. An illustration of the proposed concept for filtering application. The combined response of two clampedclamped beam resonators of hardening and softening behavior is shown. Only mono-stable response of the beams is shown here (the overlap region of the forward and backward frequency sweep). The softening behavior is induced via application of electrothermal voltage to one of the resonators. This resonator is further tuned by increasing the electrothermal voltage to combine the responses of hardening and softening to achieve a near flat pass band and sharp roll-off. 


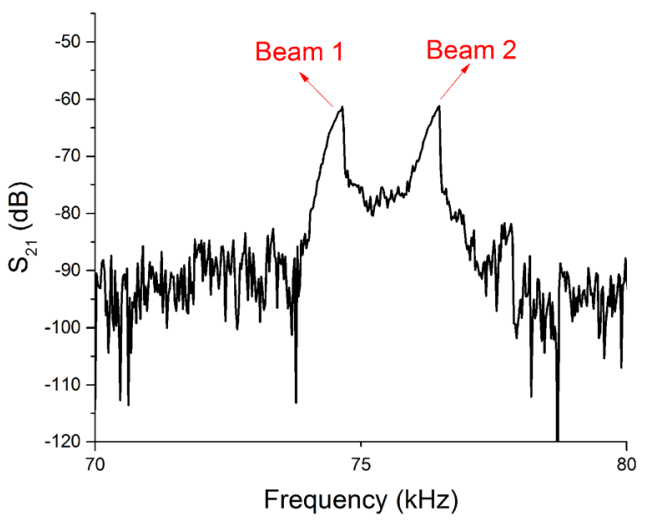

FIG. 3. The combined response of the resonators under $\mathrm{V}_{\mathrm{DC}}=55 \mathrm{~V}$ and an $\mathrm{RF}$ input of $-5 \mathrm{dBm}$. The plot shows the corresponding resonance frequencies of the two microbeams. $S_{21}$ implies two-port scattering parameter providing the forward voltage gain of the device under test, where port 1 is configured as input and port 2 as output.

the overlapping of the nonlinear skewed response peaks of the resonators. Similarly, a sharp roll-off is achieved due to the abrupt fall of amplitude at either end of the response due to the combined jumps from the hardening and softening resonance peaks on both sides of the pass band. The bandwidth of the filter can be tuned by adjusting $\mathrm{V}_{\mathrm{T}}$ applied to any one of the resonators. Furthermore, the center frequency of the filter can be tuned by applying $\mathrm{V}_{\mathrm{T}}$ independently to both resonators and accordingly adjusting their resonance frequencies.

It is worth mentioning here that the nonlinear response of the resonator differs in the forward and backward sweeps resulting in hysteresis in the response. ${ }^{4}$ Hence, the response can be mono-stable, with a unique state for a given excitation frequency, or bi-stable where two states of small and large amplitude vibration can coexist [see Figs. 5(a) and 5(b)]. Figures 5(c) and 5(d) show the experimental response of the resonators analogous to the schematics shown in Figs. 5(a) and 5(b).

Figure 5(a) shows that the combined response of both beams has a single mono-stable regime in the middle and two bi-stable regimes at each end. The forward sweep defines the edge of the mono-stable regime from the left while the backward sweep defines the edge of the mono-stable regime from the right. The mono-stable region here is shrunk by bringing the two responses close to each other to create a flat pass band [see Fig. 5(b)]. This mono-stable region defines the operating range of the filter [see Figs. 5(c) and 5(d)]. For a fixed electrostatic loading, the response outside the mono-stable region can be achieved if continuation of initial conditions is applied, through a frequency sweep for instance, where the energy from the previous state is carried into the next state. For the discrete values of input frequencies and a given electrostatic loading, the resonator ideally jumps to the frequency branch (higher state of vibration) only inside the mono-stable region and stays at the non-resonant branch (lower state of vibration) in the bi-stable region. Hence, the filter operating range is considered to be the overlapping mono-stable region only. These jumps however can be uncertain depending on the strength of the basin of attraction of each branch.

Despite the improvement of the pass band flatness and roll-off characteristics, the proposed filter needs improvements in some aspects. First, the power handling capability of the device could be low considering that a significant incoming $\mathrm{AC}$ power can widen the pass band region, thus creating uncertainty in the filter operation close to the hysteresis region. Minimizing the hysteresis regions, by controlling the nonlinearity strength, will help resolve such uncertainty. To improve understanding of the filter performance within this hysteric regime, the behavior of the filter in response to a real time broadband signal needs to be extensively studied. This is particularly needed in light of the fact that the response of the resonators in the bi-stable regime to a random excitation input is not currently totally understood. The basin of attraction analysis to reveal the strength and robustness of the two dynamical states in this regime needs be conducted. Also, the VNA may not represent accurately the behavior of a broadband signal. Hence, it is necessary to investigate the effects of the carrier signal's noise and the multi-frequency excitation, including amplitude and frequency modulated signals.

We also notice that the bi-stable region towards the left of the mono-stable regions is quite large due to the strong nonlinearity of the buckled beam [see Fig. 5(c)]. This region can be reduced by operating in areas where the beam's curvature is smaller due to the electrothermal loading. This can be achieved by tuning both beams to an optimized frequency value, where the hysteresis regions are small on each side. Nevertheless, in its current form, the region of uncertain filter behavior for a small power handling capacity will be still very close to the hysteresis regions, irrespective of the large bi-stable region to the left. This is due to the fact that a large amount of RF power will be required for the filter response signal to jump to the far left frequency branch of this bistable region [see Fig. 5(c)]. (a)

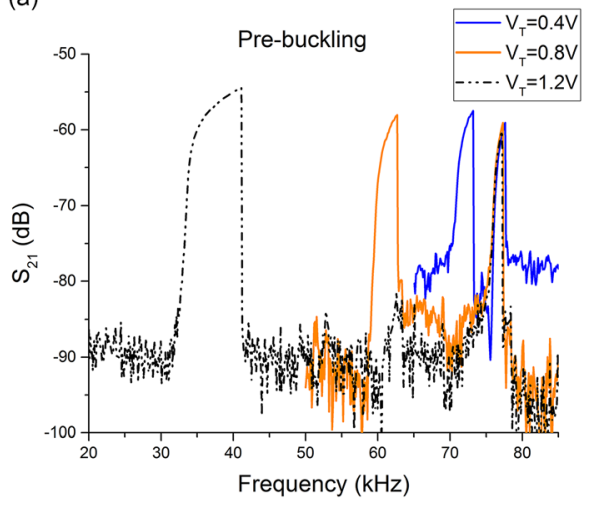

(b)

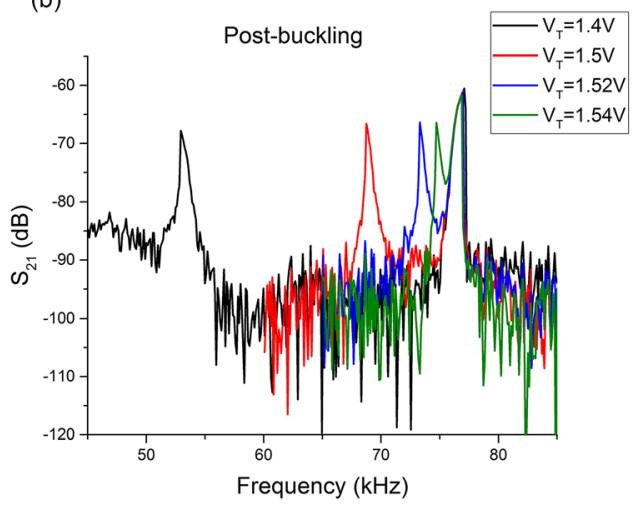

FIG. 4. The combined response of the resonators under $\mathrm{V}_{\mathrm{DC}}=55 \mathrm{~V}$, and an $\mathrm{RF}$ input of $-5 \mathrm{dBm}$ for various electrothermal voltages applied to beam 1 , (a) before buckling of beam 1 , where the increase in electrothermal voltage causes the resonance frequency to decrease while showing hardening nonlinearity, and (b) after buckling of beam 1 , where the increase in electrothermal voltage causes the resonance frequency to increase while showing softening nonlinearity. 
(a)

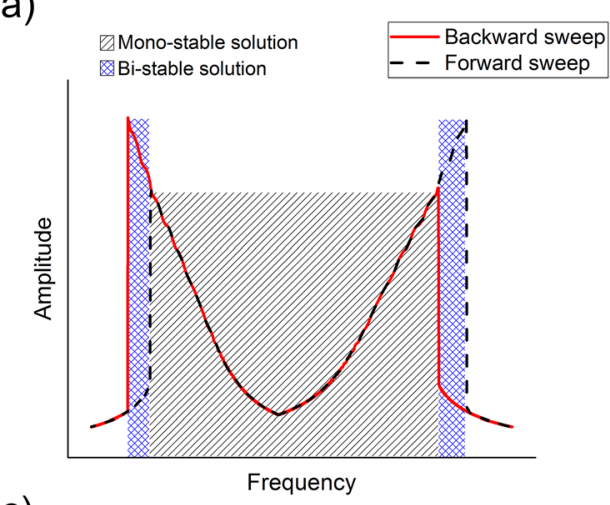

(c)

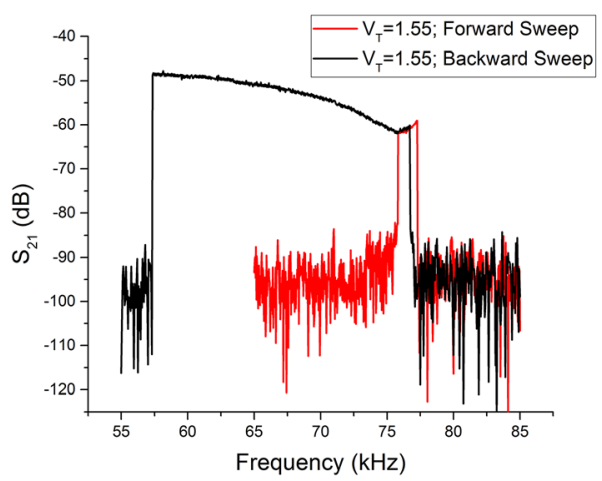

(b)

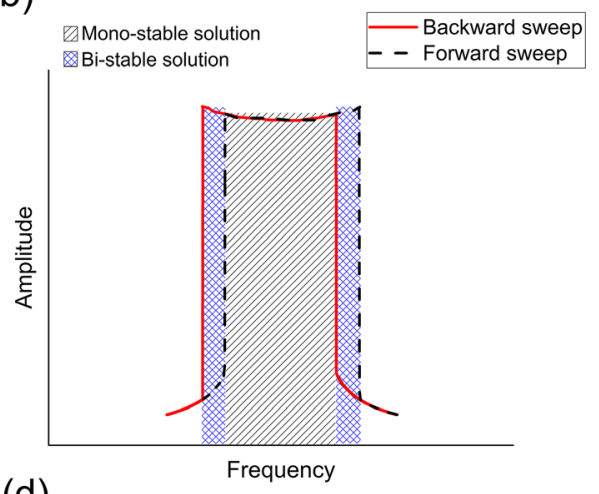

(d)

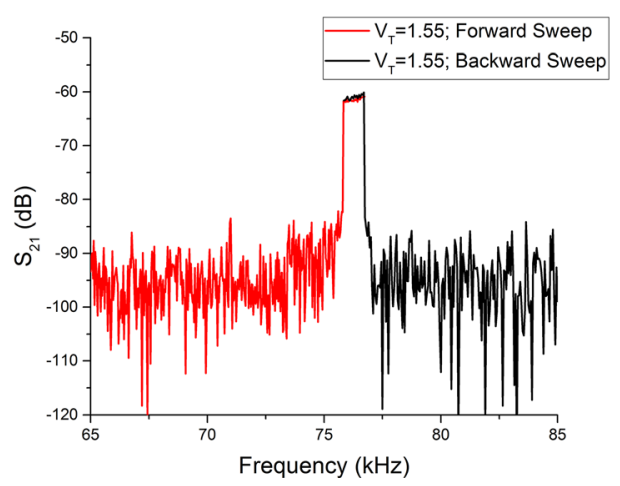

FIG. 5. (a) and (b) Schematics of the forward (dashed) and backward (solid) sweeps of the proposed filter for (a) unmerged response of the resonator and (b) merged response of the resonators. The mono-stable and bi-stable regimes of the filter response are identified and highlighted. (c) and (d) The experimental combined response of the resonators under an electrostatic loading of $\mathrm{V}_{\mathrm{DC}}=55 \mathrm{~V}$, an $\mathrm{RF}$ input of $-5 \mathrm{dBm}$, and an electrothermal voltage $\mathrm{V}_{\mathrm{T}}=1.55 \mathrm{~V}$ for (c) forward and backward sweep and (d) overlapping region of (c).
A MEMS filter is demonstrated here by combining the nonlinear softening and hardening responses from two resonators. The device is demonstrated to have a near flat pass band with sharp roll-off characteristics. One aspect that needs further investigation is to control the nonlinearity of the hysteresis region. Also, the basin of attractions analysis in the bi-stable regimes can help determine the optimal operating conditions for the filter. This is an area for future research and it can prove fruitful in developing band pass filter of improved characteristics.

This research has been supported by the King Abdullah University of Science and Technology (KAUST) research fund.

${ }^{1}$ D. N. Guerra, A. R. Bulsara, W. L. Ditto, S. Sinha, K. Murali, and P. Mohanty, Nano Lett. 10, 1168 (2010).

${ }^{2}$ A. Yao and T. Hikihara, Appl. Phy. Lett. 105, 123104 (2014).

${ }^{3}$ A. Z. Hajjaj, M. A. Hafiz, and M. I. Younis, Sci. Rep. 7, 41820 (2017).

${ }^{4}$ M. I. Younis, MEMS Linear and Nonlinear Statics and Dynamics (Springer, 2011).

${ }^{5}$ N. Kacem, S. Hentz, D. Pinto, B. Reig, and V. Nguyen, Nanotechnolgy 20, 275501 (2009).

${ }^{6}$ W. G. Conley, A. Raman, C. M. Krousgrill, and S. Mohammadi, Nano Lett. 8, 1590 (2008).

${ }^{7}$ A. Eichler, J. Moser, M. I. Dykman, and A. Bachtold, Nat. Commun. 4, 2843 (2013).

${ }^{8}$ A. Eichler, J. Moser, J. Chaste, M. Zdrojek, I. Wilson-Rae, and A. Bachtold, Nat. Nanotechol. 6, 339 (2011).

${ }^{9}$ R. B. Karabalin, M. C. Cross, and M. L. Roukes, Phys. Rev. B 79, 165309 (2009).

${ }^{10}$ C. Cassella, Y. Hui, Z. Qian, G. Hummel, and M. Rinaldi, J. Microelectromech. Syst. 25, 275 (2016).

${ }^{11}$ C. Y. Chen, M. H. Li, C. H. Chin, C. S. Li, and S. S. Li, in IEEE International Conference on Micro Electro Mechnical Systems (2014), p. 1249.

${ }^{12}$ M. M. Shalaby, M. A. Abdel Moneum, and K. Saitou, IEEE Trans. Ind. Electron. Control Instrum. 56, 1022 (2009).

${ }^{13}$ B. K. Hammad, Shock Vib. 2014, Article 939467 (2017).

${ }^{14}$ F. D. Bannon III, J. R. Clark, and C. T.-C. Nguyen, IEEE J. Solid-State Circuits 35, 512-526 (2000).
${ }^{15}$ S. Pourkamali and F. Ayazi, Sensor. Actuators, A 122, 307 (2005).

${ }^{16}$ S. Pourkamali and F. Ayazi, Sensor. Actuators A 122, 317 (2005).

${ }^{17}$ M.-H. Li, C.-Y. Chen, W.-C. Chen, and S.-S. Li, J. Microelectromech. Syst. 24, 528 (2015).

${ }^{18}$ Y. S. Lin, Y. Qian, F. Ma, Z. Liu, P. Kropelnicki, and C. Lee, Appl. Phy. Lett 102, 111908 (2013).

${ }^{19}$ X. Zou, J. Yan, and A. A. Seshia, Procedia Eng. 5, 1446 (2010).

${ }^{20}$ M. S. Hajhashemi, A. Amini, and B. Bahreyni, Sensor. Actuators A 187, 10 (2012).

${ }^{21}$ J. L. Lopez, J. Verd, A. Uranga, J. Giner, G. Murillo, F. Torres, G. Abadal, and N. Barniol, Electron Device Lett. 30, 718 (2009).

${ }^{22}$ T. L. Naing, J. N. Nilchi, R. Liu, T. O. Rocheleau, and C. T. Nguyen, in IEEE Frequency Control Symposium (FCS) (2015), p. 1.

${ }^{23}$ B. K. Hammad, E. M. Abdel-Rahman, and A. H. Nayfeh, Nonlinear Dyn. 60, 385 (2010).

${ }^{24}$ G. Piazza, P. J. Stephanou, and A. P. Pisano, J. Microelectromech. Syst. 15, 1406 (2006)

${ }^{25}$ R. C. Ruby, P. Bradley, Y. Oshmyansky, A. Chien, and J. D. Larson, IEEE Ultrason. Symp. 1, 821 (2001).

${ }^{26}$ Y. H. Cho and G. M. Rebeiz, IEEE Trans. Microwave Theory Tech. 62, 290 (2014).

${ }^{27}$ J. F. Rhoads, S. W. Shaw, K. L. Turner, and R. Baskaran, J. Vib. Acoust. 127, 423 (2005)

${ }^{28}$ S. W. Shaw, K. L. Turner, J. F. Rhoads, and R. Baskaran, in IUTAM Symposium on Chaotic Dynamics and Control of Systems and Processes in Mechanics (2005), p. 137.

${ }^{29}$ B. K. Hammad, A. H. Nayfeh, and E. M. Abdel-Rahman, J. Comp. Nonlinear Dyn. 6, 041007 (2011).

${ }^{30}$ B. K. Hammad, A. H. Nayfeh, and E. M. Abdel-Rahman, in ASME International Mechanical Engineering Congress Exposition (2008), p. 371.

${ }^{31}$ H. M. Ouakad, A. H. Nayfeh, S. Choura, E. M. Abdel-Rahman, F. Najar, and B. K. Hammad in ASME International Mechanical Engineering Congress Exposition (2008), p. 535.

${ }^{32}$ A. C. Wong and C. T. Nguyen, J. Microelectromech. Syst. 13, 100 (2004).

${ }^{33}$ F. Chen, J. Brotz, U. Arslan, C. C. Lo, T. Mukherjee, and G. K. Fedder, in Proceedings of the 18th IEEE International Conference on Micro Electro Mechnical Systems (2005), p. 24.

${ }^{34}$ S. Ilyas, K. N. Chappanda, M. A. A. Hafiz, A. Ramini, and M. I. Younis, Sensor. Actuators A 247, 368 (2016).

${ }^{35} \mathrm{~K}$. Wang and C. C. Nguyen, in IEEE International Conference on Micro Electro Mechnical Systems (1997), p. 25.

${ }^{36}$ A. Z. Hajjaj, N. Alcheikh, A. Ramini, M. A. A. Hafiz, and M. I. Younis, J. Microelectromech. Syst. 25, 440 (2016). 